\title{
Novel cross-linkers for PDMS networks for controlled and well distributed grafting of functionalities by click chemistry
}

Bahrt, Frederikke; Dimitrov, Ivaylo; Daugaard, Anders Egede; Hvilsted, Søren; Skov, Anne Ladegaard

Published in:

Polymer Chemistry

Link to article, DOI:

$10.1039 / \mathrm{c} 2 \mathrm{py} 20966 \mathrm{~g}$

Publication date:

2013

Document Version

Publisher's PDF, also known as Version of record

Link back to DTU Orbit

Citation (APA):

Bahrt, F., Dimitrov, I., Daugaard, A. E., Hvilsted, S., \& Skov, A. L. (2013). Novel cross-linkers for PDMS

networks for controlled and well distributed grafting of functionalities by click chemistry. Polymer Chemistry, 4(5), 1700-1707. https://doi.org/10.1039/c2py20966g

\section{General rights}

Copyright and moral rights for the publications made accessible in the public portal are retained by the authors and/or other copyright owners and it is a condition of accessing publications that users recognise and abide by the legal requirements associated with these rights.

- Users may download and print one copy of any publication from the public portal for the purpose of private study or research.

- You may not further distribute the material or use it for any profit-making activity or commercial gain

- You may freely distribute the URL identifying the publication in the public portal 


\title{
Polymer Chemistry
}

\section{Novel cross-linkers for PDMS networks for controlled and well distributed grafting of functionalities by click chemistry $\dagger$}

\author{
Frederikke Bahrt Madsen, Ivaylo Dimitrov, Anders Egede Daugaard, Søren Hvilsted \\ and Anne Ladegaard Skov*
}

\begin{abstract}
An azide-containing, trifunctional vinyl cross-linker for silicone networks has been synthesized. The crosslinker has through $\mathrm{Cu}(\mathrm{I})$ catalyzed 1,3-cycloaddition been reacted with six different alkyne-containing chemical groups which each possess a particular functionality. The functional cross-linkers have been utilized to prepare novel polydimethylsiloxane (PDMS) networks. All functional cross-linkers were successfully incorporated into the networks and were demonstrated to be well distributed within the PDMS films. This was substantiated by fluorescence microscopy of a film prepared with the 4-methylumbelliferone containing cross-linker. TGA showed that a ferrocene functionality increased the thermal degradation temperature of PDMS. It was furthermore shown that the incorporation of only $0.25 \mathrm{wt} \%$ of the push-pull dipole, ethynyl-4-nitrobenzene, increased the dielectric permittivity of PDMS by $35 \%$. The contact angle of PDMS films was increased from $108^{\circ}$ to $116^{\circ}$ by the introduction of a small poly(pentafluorostyrene) chain. Finally, 17 $\alpha$-ethynyl-1,3,5(10)-estratriene-3,17 $\beta$-diol and 1-ethynyl-3,5bis(trifluoromethyl)benzene were incorporated as examples of other functional groups.
\end{abstract}

Received 14th November 2012
Accepted 15th December 2012

DOI: $10.1039 /$ c2py20966g

www.rsc.org/polymers envisioned to be performed by covalent attachment of functional molecules to the network, which would provide a matrix with well distributed modifications that prevent leakage of the functional moieties from the PDMS network during use. Modification of the PDMS network by covalent grafting of functionality will change the properties and improve the applicability of PDMS within many areas such as electrical, optical and biomedical areas. The modification could furthermore improve properties such as oil and solvent resistance ${ }^{7}$ and current release problems encountered for thin PDMS films where the release forces between PDMS and the substrate easily exceed the tear strength of the material since the excellent wetting properties of PDMS may lead to strong substrate-PDMS interactions. $^{8}$

The cross-linking of PDMS into an elastomer is frequently achieved by hydrosilylation where vinyl groups of one component react with hydrosilane groups of the other component in a platinum catalyzed reaction. In this work, a novel silicone compatible vinyl cross-linker containing an azide functionality is introduced. The vinyl groups allow for cross-linking reactions with hydride-terminated PDMS molecules whereas the azide group allows for modification of the PDMS network either before or after network cross-linking. The azide moiety opens up for click reactions, in this case the copper-catalyzed cycloaddition of an azide group and an alkyne (CuAAC) forming a 1,4-disubstituted-1,2,3-triazole. ${ }^{9-12}$ Click chemistry has previously been demonstrated to be successful for functionalization of polymers in general ${ }^{13-15}$ and in combination with 
polysiloxanes in particular. ${ }^{\mathbf{1 6}-\mathbf{1 9}}$ The azide group on the crosslinker therefore enables reactions with various types of alkyne functional molecules and thereby significantly widens the functionality that can be introduced into PDMS elastomers. Therefore a number of different functionalities are elaborated. Introduction of 4-methylumbelliferone will provide the PDMS network with a fluorescent tag at the cross-linking site that can not only be used to visualize the distribution of incorporated functionality but also create luminescent PDMS films. A pushpull dipole that potentially enhances the dielectric permittivity of the silicone elastomer can be inserted from an ethynyl-4nitrobenzene and thus improve the dielectric elastomer performance. ${ }^{\mathbf{2 0}}$ The incorporation of ferrocene will provide PDMS films with potential applications within the optical, magnetic or electronic field ${ }^{21}$ and within the area of cancer research as certain ferricenium complexes are known to show anti-tumor activity. ${ }^{22}$ Ferrocene has also recently been positioned on surfaces of nanoparticles ${ }^{23}$ or single-walled carbon nanotubes $^{\mathbf{2 4}}$ by application of click chemistry and ethynylferrocene. Different kinds of drugs or therapeutics for biomedical applications will also be possible by the introduction of an estradiol functionality. ${ }^{25}$ Recently, it has been shown that introduction of trifluoromethyl groups improve the oil and solvent resistance of PDMS films. ${ }^{7}$ With this in mind bis(trifluoromethyl)phenyl and a short poly(pentafluorostyrene) chain will be inserted into the PDMS network. Also possible improvements of the release properties of such fluorine containing PDMS films during processing will be investigated. An important property of the system is that the alkyne-functional molecule in principle can be clicked onto the azide cross-linker before or after the cross-linking reaction that leads to the PDMS network.

Here we present the preparation and characterization of novel PDMS networks containing the elaborated functionalities using the pre-cross-linking approach with the series of new silicone compatible cross-linkers. The pre-cross-linking procedure avoids the step of swelling the PDMS network for successful click reactions and furthermore allows for comprehensive characterization of the individual functional crosslinkers.

\section{Experimental}

\section{Materials and methods}

Hydride-terminated PDMS, DMS-H31 $\left(\bar{M}_{\mathrm{n}}=28000 \mathrm{~g} \mathrm{~mol}^{-1}\right)$ and a vinyl functional PDMS cross-linker, VDT-431 $\left(\bar{M}_{\mathrm{n}}=28000\right.$ $\mathrm{g} \mathrm{mol}^{-1}$, 16-functional) were acquired from Gelest Inc. The platinum cyclovinylmethyl siloxane complex catalyst (511) was purchased from Hanse Chemie AG. All other chemicals were acquired from Aldrich and used as received unless otherwise specified.

FTIR was conducted on a PerkinElmer Spectrum One model 2000 Fourier Transform Infrared apparatus equipped with a universal attenuated total reflection accessory on a ZnSe-diamond composite. Spectra were recorded in the range of 4000$650 \mathrm{~cm}^{-1}$ with $4 \mathrm{~cm}^{-1}$ resolution and 16 scans. ${ }^{1} \mathrm{H}$ - and ${ }^{13} \mathrm{C}$ NMR experiments were performed on a Bruker $250 \mathrm{MHz}$ spectrometer. Solid state ${ }^{13} \mathrm{C}-\mathrm{NMR}$ was recorded on a Bruker Avance $500 \mathrm{MHz}$ spectrometer. Thermogravimetric analysis (TGA) was performed on a Q500 from TA Instruments in a nitrogen atmosphere with a heating rate of $10{ }^{\circ} \mathrm{C} \mathrm{min}^{-1}$ from RT to $950{ }^{\circ} \mathrm{C}$. Differential scanning calorimetry (DSC) measurements were performed on a DSC Q1000 from TA Instruments. The thermal analyses were performed with a heating and cooling rate of $10{ }^{\circ} \mathrm{C} \min ^{-1}$ from $25-200{ }^{\circ} \mathrm{C}$. Mechanical characterization of the prepared films was performed with a TA Instruments 2000 Rheometer set to a controlled strain mode at $1 \%$ strain, which was ensured to be within the linear viscoelastic regime. The measurements were performed at RT with a parallel plate geometry of $25 \mathrm{~mm}$ in the frequency range of 100$0.01 \mathrm{~Hz}$. Optical characterization was performed on a Leica DMLB microscope with a Olympus Highlight 2000 light source. Fluorescence intensity measurements were performed on a BMG Labtech POLARstar Omega (excitation $355 \mathrm{~nm}$, emission $460 \mathrm{~nm}$ ). Size-exclusion chromatography (SEC) was performed on a Viscotek GPCmax VE-2001 instrument equipped with a Viscotek TrisEC Model 302 triple detector using two PLgel mixed-D columns from Polymer Laboratories. Samples were run

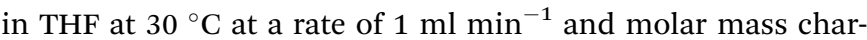
acteristics were calculated using polystyrene standards. Dielectric relaxation spectroscopy (DRS) was performed on a Novocontrol Alpha-A high performance frequency analyzer. Contact angle measurements with water were conducted on a Dataphysics OCA 20. A drop of $6 \mu$ l was placed on the surface of the film with the needle inside the drop. The drop was expanded at a rate of $0.125 \mathrm{ml} \mathrm{s}^{-1}$. All reported contact angles are an average of 3 measurements on 3 drops.

\section{Synthesis}

All reactions were carried out under a nitrogen atmosphere.

Tris(allyloxy)(3-bromopropyl)silane (1). Allyl alcohol (4.24 g, $73.11 \mathrm{mmol})$ was dissolved in dry THF $(120 \mathrm{ml})$ in a $250 \mathrm{ml} \mathrm{2-}$ necked round bottomed flask. The mixture was cooled to $0{ }^{\circ} \mathrm{C}$ in an ice bath and triethylamine (9.87 g, $97.5 \mathrm{mmol}$ ) was added dropwise. (3-Bromopropyl)trichlorosilane (5 g, $19.5 \mathrm{mmol}$ ) was then added dropwise to the reaction mixture. The reaction was carried out for 17 hours at RT. The reaction mixture was filtered and the solvent and excess allyl alcohol and triethylamine were removed in vacuo. The product was obtained in the form of a light yellow oil (5.35 g, 86\%). IR ( $\left.\mathrm{cm}^{-1}\right): 2870$ (C-H stretch); 1645 (C=C stretch); 1070 (C-O stretch); 1035 (Si-O stretch). ${ }^{1} \mathrm{H}$ NMR $\left(\mathrm{CDCl}_{3}, \delta_{\mathrm{H}}, \mathrm{ppm}\right): 0.81\left(\mathrm{~m}, 2 \mathrm{H}, \mathrm{CH}_{2}-\mathrm{Si}\right) ; 1.98\left(\mathrm{~m}, 2 \mathrm{H}, \mathrm{CH}_{2}-\mathrm{CH}_{2}-\right.$ $\left.\mathrm{CH}_{2}\right), 3.40\left(\mathrm{t},{ }^{3} \mathrm{~J}=5.6 \mathrm{~Hz}, 2 \mathrm{H}, \mathrm{Br}-\mathrm{CH}_{2}\right), 4.30\left(\mathrm{~m}, 6 \mathrm{H}, \mathrm{O}-\mathrm{CH}_{2}\right), 5.12$ $\left(\mathrm{dd},{ }^{3} J=8.6 \mathrm{~Hz}\right.$ and $\left.{ }^{2} J=1.25 \mathrm{~Hz}, 3 \mathrm{H}, \mathrm{CH}=\mathrm{C}\right), 5.27\left(\mathrm{dd}, 3 \mathrm{H},{ }^{3} J=\right.$ $14.25 \mathrm{~Hz}$ and $\left.{ }^{2} J=1.5 \mathrm{~Hz}, \mathrm{CH}=\mathrm{C}\right), 5.92\left(\mathrm{~m}, 3 \mathrm{H},=\mathrm{CH}-\mathrm{CH}_{2}\right) \cdot{ }^{13} \mathrm{C}-$ NMR ( $\left.\mathrm{CDCl}_{3}, \delta_{\mathrm{C}}, \mathrm{ppm}\right): 9.55$ (d); 26.48 (e); 36.51 (f); 63.60 (c); 114.84 (a); 136.30 (b).

Tris(allyloxy)(3-azidopropyl)silane (2). 1 (5.16 g, $16.06 \mathrm{mmol}$ ) and $\mathrm{NaN}_{3}(1.39 \mathrm{~g}, 21.41 \mathrm{mmol})$ were dissolved in DMF $(50 \mathrm{ml})$ in a $250 \mathrm{ml}$ 2-necked round bottomed flask. The reaction mixture was stirred at RT for 17 hours. The reaction mixture was then quenched with $\mathrm{H}_{2} \mathrm{O}(70 \mathrm{ml})$ and extracted with ethyl acetate $(3 \times$ $70 \mathrm{ml}$ ). The organic phases were combined and washed with 
$\mathrm{H}_{2} \mathrm{O}(3 \times 70 \mathrm{ml})$ and brine $(1 \times 70 \mathrm{ml})$, dried with $\mathrm{MgSO}_{4}$, filtered and concentrated in vacuo to give the product in the form of an orange-yellow oil $(3.91 \mathrm{~g}, 86 \%)$. IR $\left(\mathrm{cm}^{-1}\right): 2870(\mathrm{C}-\mathrm{H}$ stretch); 2095 ( $-\mathrm{N}_{3}$ stretch); $1640(\mathrm{C}=\mathrm{C}$ stretch); 1070 (C-O stretch); 1035 (Si-O stretch). ${ }^{1} \mathrm{H}$ NMR $\left(\mathrm{CDCl}_{3}, \delta_{\mathrm{H}}, \mathrm{ppm}\right): 0.74(\mathrm{~m}$, $\left.2 \mathrm{H}, \mathrm{CH}_{2}-\mathrm{Si}\right), 1.71\left(\mathrm{~m}, 2 \mathrm{H}, \mathrm{CH}_{2}-\mathrm{CH}_{2}-\mathrm{CH}_{2}\right), 3.26\left(\mathrm{t},{ }^{3} \mathrm{~J}=5.75 \mathrm{~Hz}\right.$, $\left.2 \mathrm{H}, \mathrm{N}_{3}-\mathrm{CH}_{2}\right), 4.30\left(\mathrm{~m}, 6 \mathrm{H}, \mathrm{O}-\mathrm{CH}_{2}\right), 5.12\left(\mathrm{dd},{ }^{3} J=8.5 \mathrm{~Hz}\right.$ and ${ }^{2} J=$ $1.25 \mathrm{~Hz}, 3 \mathrm{H}, \mathrm{CH}=\mathrm{C}), 5.27\left(\mathrm{dd},{ }^{3} J=14.5 \mathrm{~Hz}\right.$ and ${ }^{2} J=1.75 \mathrm{~Hz}, 3 \mathrm{H}$, $\mathrm{CH}=\mathrm{C}), 5.92\left(\mathrm{~m}, 3 \mathrm{H},=\mathrm{CH}-\mathrm{CH}_{2}\right) \cdot{ }^{13} \mathrm{C}-\mathrm{NMR}\left(\mathrm{CDCl}_{3}, \delta_{\mathrm{C}}, \mathrm{ppm}\right)$ : 7.60 (d); 27.47 (e); 53.69 (f); 63.58 (c); 114.77 (a); 136.29 (b).

4-Methyl-7-(prop-2-yn-1-yloxy)-2H-chromen-2-one (3). 4Methylumbelliferone ( $3 \mathrm{~g}, 17,0 \mathrm{mmol})$ and $\mathrm{K}_{2} \mathrm{CO}_{3}(2.04 \mathrm{~g}, 20$ $\mathrm{mmol})$ were dissolved in acetone $(85 \mathrm{ml})$. Propargyl bromide $(2.43 \mathrm{~g}, 20 \mathrm{mmol})$ was added at a rate of $0.06 \mathrm{ml} \mathrm{min}^{-1}$. The reaction was carried out under reflux for 24 hours. The reaction mixture was then quenched with $\mathrm{H}_{2} \mathrm{O}(80 \mathrm{ml})$ and subsequently extracted 3 times with ethyl acetate. The organic phases were combined and washed with $\mathrm{H}_{2} \mathrm{O}(3 \times 80 \mathrm{ml})$ and brine $(1 \times 80$ $\mathrm{ml}$ ), dried with $\mathrm{MgSO}_{4}$, filtered and concentrated in vacuo. The crude was recrystallized in an ethyl acetate-heptane mixture $(1: 0.75)$ to give the product in the form of a white solid in quantitative yield. IR $\left(\mathrm{cm}^{-1}\right): 3305(\mathrm{C} \equiv \mathrm{C}-\mathrm{H}$ stretch); $1720(\mathrm{O}-$ $\mathrm{C}=\mathrm{O}$ stretch $) ; 1605(\mathrm{C}=\mathrm{C}$ stretch $) .{ }^{1} \mathrm{H} \mathrm{NMR}\left(\mathrm{CDCl}_{3}, \delta_{\mathrm{H}}, \mathrm{ppm}\right)$ : $2.40\left(\mathrm{~s}, 3 \mathrm{H}, \mathrm{Ar}-\mathrm{CH}_{3}\right), 2.57(\mathrm{~s}, 1 \mathrm{H}, \mathrm{C} \equiv \mathrm{C}-H), 4.76\left(\mathrm{~d},{ }^{4} \mathrm{~J}=2 \mathrm{~Hz}, 2 \mathrm{H}\right.$, $\left.\mathrm{O}-\mathrm{CH}_{2}-\right), 6.16(\mathrm{~s}, 1 \mathrm{H}, \mathrm{O}=\mathrm{C}-\mathrm{CH}-), 6.95(\mathrm{~m}, 2 \mathrm{H}, \mathrm{Ar}-\mathrm{H}), 7.52(\mathrm{~m}$, $1 \mathrm{H}, \mathrm{Ar}-H) .{ }^{13} \mathrm{C}-\mathrm{NMR}\left(\mathrm{CDCl}_{3}, \delta_{\mathrm{C}}, \mathrm{ppm}\right): 18.58(\mathrm{~s}) ; 56.09(\mathrm{i}) ; 76.43$ (g); 77.34 (h); 102.07 (o); $112.31(\mathrm{q}) ; 112.59(\mathrm{k}) ; 114.16(\mathrm{~m})$; 125.56 (l); 152.56 (n); 154.93 (p); 160.27 (j); 160.99 (r).

General procedure for click reactions, 4-methyl-7-((1-(3-(tris(allyloxy)silyl)propyl)-1H-1,2,3-triazol-4-yl)methoxy)-2H-chromen2-one (4). 2 (3.01 g, $10.6 \mathrm{mmol})$ and 3 (2.17 g, $10.0 \mathrm{mmol})$ were dissolved in THF (50 ml) in a $250 \mathrm{ml}$ 2-necked round bottomed flask. CuI (0.206 g, $10.6 \mathrm{mmol}$ ) was subsequently added and $\mathrm{Et}_{3} \mathrm{~N}(1.61 \mathrm{~g}, 15.9 \mathrm{mmol})$ was added dropwise. The reaction was carried out at RT for 17 hours. The reaction mixture was extracted with ethyl acetate and washed with $\mathrm{H}_{2} \mathrm{O}(3 \times 40 \mathrm{ml})$ and brine $(1 \times 40 \mathrm{ml})$, dried with $\mathrm{MgSO}_{4}$, filtered and concentrated in vacuo to give the product in the form of a light grey oil $(2.66 \mathrm{~g}, 53 \%)$. IR $\left(\mathrm{cm}^{-1}\right): 2935(\mathrm{C}-\mathrm{H}$ stretch$) ; 1715(\mathrm{O}-\mathrm{C}=\mathrm{O}$ stretch); 1610 (C=C stretch); 1070 (C-O stretch); 1035 ( $\mathrm{Si}-\mathrm{O}$ stretch). ${ }^{1} \mathrm{H}$ NMR $\left(\mathrm{CDCl}_{3}, \delta_{\mathrm{H}}, \mathrm{ppm}\right): 0.60\left(\mathrm{~m}, 2 \mathrm{H}, \mathrm{CH}_{2}-\mathrm{Si}\right), 2.05$ $\left(\mathrm{m}, 2 \mathrm{H}, \mathrm{CH}_{2}-\mathrm{CH}_{2}-\mathrm{CH}_{2}\right), 2.40\left(\mathrm{~s}, 3 \mathrm{H}, \mathrm{Ar}-\mathrm{CH}_{3}\right), 4.28(\mathrm{~m}, 2 \mathrm{H}, \mathrm{N}-$ $\left.\mathrm{CH}_{2}-\right), 4.37\left(\mathrm{~m}, 6 \mathrm{H}, \mathrm{O}-\mathrm{CH}_{2}-\right), 5.11$ (dd, ${ }^{3} \mathrm{~J}=8.5 \mathrm{~Hz}$ and ${ }^{2} \mathrm{~J}=1.25$ $\mathrm{Hz}, 3 \mathrm{H}, \mathrm{CH}_{2}=\mathrm{C}$ ), 5.25 (s, 2H, O-C $\mathrm{H}_{2}-\mathrm{C}-\mathrm{N}$ ), 5.26 (dd, ${ }^{3} \mathrm{~J}=14.25$ $\mathrm{Hz}$ and $\left.{ }^{2} J=1.5 \mathrm{~Hz}, 3 \mathrm{H}, \mathrm{CH}=\mathrm{C}\right), 5.92\left(\mathrm{~m}, 3 \mathrm{H},=\mathrm{CH}-\mathrm{CH}_{2}\right), 6.14$ (s, $1 \mathrm{H}, \mathrm{O}=\mathrm{C}-\mathrm{CH}-)$, $6.95(\mathrm{~m}, 2 \mathrm{H}, \mathrm{Ar}-H), 7.50(\mathrm{~m}, 1 \mathrm{H}, \mathrm{Ar}-H), 7.65$ $(\mathrm{s}, 1 \mathrm{H},-\mathrm{C}=\mathrm{CH}-\mathrm{N}-) .{ }^{13} \mathrm{C}-\mathrm{NMR}\left(\mathrm{CDCl}_{3}, \delta_{\mathrm{C}}, \mathrm{ppm}\right): 7.39$ (d); 18.54 (s); 23.93 (e); 52.39 (f); 62.20 (i); 63.70 (c); 101.98 (o); 112.08 (q); 112.27 (k); 113.89 (l); 114.90 (a); 122.95 (m); 125.60 (g); 136.10 (b), 142.69 (h); 152.39 (n); 154.99 (p); 161.02 (j); 161.07 (r).

4-Ferrocene-1-(3-(tris(allyloxy)silyl)propyl)-1H-1,2,3-triazole (5). The product was prepared according to the general click procedure using $2(0.204 \mathrm{~g}, 0.71 \mathrm{mmol})$ and ethynylferrocene (0.145 g, $0.67 \mathrm{mmol})$, CuI $(0.0134 \mathrm{~g}, 0.071 \mathrm{mmol})$ and $\mathrm{Et}_{3} \mathrm{~N}$ $(0.107 \mathrm{~g}, 1.06 \mathrm{mmol})$ to give a brown oil $(0.31 \mathrm{~g}, 94 \%)$. IR $\left(\mathrm{cm}^{-1}\right)$ : 2955-2870 (C-H stretch); 1590 ( $\mathrm{C}=\mathrm{C}$ ring stretch); $1430(\mathrm{C}=\mathrm{C}$ ring stretch); 1070 (C-O stretch); 1035 (Si-O stretch). ${ }^{1} \mathrm{H}$ NMR
$\left(\mathrm{CDCl}_{3}, \delta_{\mathrm{H}}, \mathrm{ppm}\right): 0.68\left(\mathrm{~m}, 2 \mathrm{H}, \mathrm{CH}_{2}-\mathrm{Si}\right), 2.07\left(\mathrm{~m}, 2 \mathrm{H}, \mathrm{CH}_{2}-\mathrm{CH}_{2}-\right.$ $\left.\mathrm{CH}_{2}\right), 4.07\left(\mathrm{~m}, 2 \mathrm{H}, \mathrm{N}-\mathrm{CH}_{2}-\right), 4.2-4.25(\mathrm{~m}, 8 \mathrm{H}, \mathrm{Ar}-\mathrm{H}), 4.30(\mathrm{~m}$, $\left.6 \mathrm{H}, \mathrm{O}-\mathrm{CH}_{2}-\right), 4.72(\mathrm{~m}, 1 \mathrm{H}, \mathrm{Ar}-\mathrm{H}), 5.12\left(\mathrm{dd},{ }^{3} \mathrm{~J}=8.75 \mathrm{~Hz}\right.$ and ${ }^{2} \mathrm{~J}=$ $1.25 \mathrm{~Hz}, 3 \mathrm{H}, \mathrm{C} H=\mathrm{C}), 5.28\left(\mathrm{dd},{ }^{3} J=14.25 \mathrm{~Hz}\right.$ and ${ }^{2} J=1.25 \mathrm{~Hz}$, $3 \mathrm{H}, \mathrm{CH}=\mathrm{C}), 5.92\left(\mathrm{~m}, 3 \mathrm{H},=\mathrm{CH}-\mathrm{CH}_{2}\right), 7.46(\mathrm{~s}, 1 \mathrm{H},-\mathrm{C}=\mathrm{CH}-\mathrm{N}-)$. ${ }^{13} \mathrm{C}-\mathrm{NMR}\left(\mathrm{CDCl}_{3}, \delta_{\mathrm{C}}, \mathrm{ppm}\right): 7.40$ (d); 24.32 (e); 52.21 (f); 63.73 (c); 66.52 (k); 68.51 (j); 69.50 (i); 114.92 (a); 118.83 (g); 136.16 (b); $143.46(\mathrm{~h})$.

4-(4-Nitrophenyl)-1-(3-(tris(allyloxy)silyl)propyl)-1H-1,2,3-triazole (6). The product was prepared according to the general click procedure using $2(0.203 \mathrm{~g}, 0.72 \mathrm{mmol})$, 1-ethynyl-4-nitrobenzene $(0.103 \mathrm{~g}, 0.68 \mathrm{mmol})$, CuI $(0.0137 \mathrm{~g}, 0.072 \mathrm{mmol})$ and $\mathrm{Et}_{3} \mathrm{~N}(0.109 \mathrm{~g}, 1.07 \mathrm{mmol})$ to give a light yellow oil which crystallized upon cooling $(0.156 \mathrm{~g}, 54 \%) . M_{\mathrm{p}}: 69^{\circ} \mathrm{C}$. IR $\left(\mathrm{cm}^{-1}\right): 2915-$ 2860 (C-H stretch); 1645 (C=C stretch); 1605 (aromatic $\mathrm{C}=\mathrm{C}$ stretch); 1505 ( $\mathrm{N}=\mathrm{O}$ asymmetric stretch); 1460 (aromatic $\mathrm{C}=\mathrm{C}$ stretch); 1340 ( $\mathrm{N}=\mathrm{O}$ symmetric stretch); 1075 (C-O stretch); 1035 (Si-O stretch). ${ }^{1} \mathrm{H}$ NMR $\left(\mathrm{CDCl}_{3}, \delta_{\mathrm{H}}, \mathrm{ppm}\right): 0.71\left(\mathrm{~m}, 2 \mathrm{H}, \mathrm{CH}_{2}-\right.$ $\mathrm{Si}), 2.11\left(\mathrm{~m}, 2 \mathrm{H}, \mathrm{CH}_{2}-\mathrm{CH}_{2}-\mathrm{CH}_{2}\right), 4.31\left(\mathrm{~m}, 2 \mathrm{H}, \mathrm{N}-\mathrm{CH}_{2}-\right), 4.44(\mathrm{~m}$, $\left.6 \mathrm{H}, \mathrm{O}-\mathrm{CH}_{2}-\right), 5.13\left(\mathrm{dd},{ }^{3} \mathrm{~J}=8.75 \mathrm{~Hz}\right.$ and ${ }^{2} \mathrm{~J}=1.25 \mathrm{~Hz}, 3 \mathrm{H}, \mathrm{CH}=$ C), $5.28\left(\mathrm{dd},{ }^{3} J=14.25 \mathrm{~Hz}\right.$ and $\left.{ }^{2} J=1.25 \mathrm{~Hz}, 3 \mathrm{H}, \mathrm{CH}=\mathrm{C}\right), 5.92(\mathrm{~m}$, $\left.3 \mathrm{H},=\mathrm{CH}-\mathrm{CH}_{2}\right), 7.91-8.00(\mathrm{~m}, 2 \mathrm{H}, \mathrm{Ar}-\mathrm{H}), 8.95\left(\mathrm{~m}, 2 \mathrm{H}, \mathrm{NO}_{2}-\mathrm{Ar}-\right.$ $H), 8.30(\mathrm{~s}, 1 \mathrm{H},-\mathrm{C}=\mathrm{CH}-\mathrm{N}-) .{ }^{13} \mathrm{C}-\mathrm{NMR}\left(\mathrm{CDCl}_{3}, \delta_{\mathrm{C}}, \mathrm{ppm}\right): 7.46(\mathrm{~d})$; 24.04 (e); 52.56 (f); 63.82 (c); 115.03 (a); 121.19 (g); 124.27 (k); 126.04 (j); 136.16 (b); 136.96 (i); 145.39 (l); 147.21 (h).

4-(3,5-Bis(trifluoromethyl)phenyl)-1-(3-(tris(allyloxy)silyl)propyl)-1H-1,2,3-triazole (7). The product was prepared according to the general click procedure using $2(0.200 \mathrm{~g}, 0.706$ $\mathrm{mmol})$, and 1-ethynyl-3,5-bis(trifluoromethyl)benzene $(0.17 \mathrm{~g}$, $0.67 \mathrm{mmol})$, $\mathrm{CuI}(0.0138 \mathrm{~g}, 0.071 \mathrm{mmol})$ and $\mathrm{Et}_{3} \mathrm{~N}(0.107 \mathrm{~g}, 1.06$ $\mathrm{mmol})$ at $45{ }^{\circ} \mathrm{C}$ for 24 hours. Azide functional Merrifield resin $(0.150 \mathrm{~g}, 0.21 \mathrm{mmol})^{26}$ was added towards the end of the click reaction in order to remove excess 1-ethynyl-3,5-bis(trifluoromethyl)benzene. This reaction was carried out at $45^{\circ} \mathrm{C}$ for 17 hours. The reaction mixture was thereafter filtered and extracted with ethyl acetate $(3 \times 60 \mathrm{ml})$ and washed with $\mathrm{H}_{2} \mathrm{O}(3$ $\times 60 \mathrm{ml}$ ), dried with $\mathrm{MgSO}_{4}$, filtered and concentrated in vacuo to give the product in the form of an orange-brown oil $(0.28 \mathrm{~g}$, 80\%). IR ( $\left.\mathrm{cm}^{-1}\right)$ : 2930-2870 (C-H stretch); 1615 (aromatic $\mathrm{C}=\mathrm{C}$ stretch); 1465 (aromatic $\mathrm{C}=\mathrm{C}$ stretch); 1275 (C-F stretch), 1170 (C-F stretch), 1125 (C-F stretch), 1085 (C-O stretch); 1035 (Si-O stretch). ${ }^{1} \mathrm{H}$ NMR $\left(\mathrm{CDCl}_{3}, \delta_{\mathrm{H}}, \mathrm{ppm}\right): 0.71\left(\mathrm{~m}, 2 \mathrm{H}, \mathrm{CH}_{2}-\mathrm{Si}\right), 2.11$ $\left(\mathrm{m}, 2 \mathrm{H}, \mathrm{CH}_{2}-\mathrm{CH}_{2}-\mathrm{CH}_{2}\right), 4.31\left(\mathrm{~m}, 2 \mathrm{H}, \mathrm{N}-\mathrm{CH}_{2}-\right), 4.45(\mathrm{~m}, 6 \mathrm{H}, \mathrm{O}-$ $\left.\mathrm{CH}_{2}-\right), 5.12\left(\mathrm{dd},{ }^{3} \mathrm{~J}=8.75 \mathrm{~Hz}\right.$ and $\left.{ }^{2} \mathrm{~J}=1.25 \mathrm{~Hz}, 3 \mathrm{H}, \mathrm{CH}=\mathrm{C}\right), 5.27$ (dd, ${ }^{3} J=14.25 \mathrm{~Hz}$ and $\left.{ }^{2} J=1.5 \mathrm{~Hz}, 3 \mathrm{H}, \mathrm{CH}=\mathrm{C}\right), 5.93(\mathrm{~m}, 3 \mathrm{H},=$ $\left.\mathrm{CH}-\mathrm{CH}_{2}\right), 7.92(\mathrm{~s}, 1 \mathrm{H}, \mathrm{Ar}-\mathrm{H}), 7.94(\mathrm{~s}, 1 \mathrm{H},-\mathrm{C}=\mathrm{CH}-\mathrm{N}-), 8.28(\mathrm{~s}$, $2 \mathrm{H}, \mathrm{Ar}-H) .{ }^{13} \mathrm{C}-\mathrm{NMR}\left(\mathrm{CDCl}_{3}, \delta_{\mathrm{C}}, \mathrm{ppm}\right): 7.45$ (d); 24.07 (e); 52.59 (f); 63.74 (c); 114.96 (a); 120.78 (g); 121.55 (l); 125.54 (m); 132.03 (i); 132.47 (j); 132.89 (k); 136.27 (b); 144.93 (h).

4-(1,2,5(10)-Estratriene-1,17 $\beta$-diol)-1-(3-(tris(allyloxy)silyl)propyl)-1H-1,2,3-triazole (8). The product was prepared according to the general click procedure using $2(0.197 \mathrm{~g}, 0.71$ $\mathrm{mmol}), \quad 17 \alpha$-ethynyl-1,3,5(10)-estratriene-3,17 $\beta$-diol $(0.203 \mathrm{~g}$, $0.67 \mathrm{mmol}), \mathrm{CuI}(0.0134 \mathrm{~g}, 0.074 \mathrm{mmol})$ and $\mathrm{Et}_{3} \mathrm{~N}(0.107 \mathrm{~g}, 1.06$ $\mathrm{mmol}$ ) at RT for 17 hours then at $45{ }^{\circ} \mathrm{C}$ for 24 hours to give a light yellow oil $(0.373 \mathrm{~g}, 96 \%)$. IR $\left(\mathrm{cm}^{-1}\right)$ : 3310 (O-H stretch); 2930-2870 (C-H stretch); 1610 (aromatic $\mathrm{C}=\mathrm{C}$ stretch), 1455 
(aromatic $\mathrm{C}=\mathrm{C}$ stretch), 1055 (C-O stretch); 1035 ( $\mathrm{Si}-\mathrm{O}$ stretch). ${ }^{1} \mathrm{H}$ NMR $\left(\mathrm{CDCl}_{3}, \delta_{\mathrm{H}}, \mathrm{ppm}\right): 0.68\left(\mathrm{~m}, 2 \mathrm{H}, \mathrm{CH}_{2}-\mathrm{Si}\right), 1.04(\mathrm{~s}, 3 \mathrm{H}$, $\left.-\mathrm{CH}_{3}\right), 1.41-2.82(\mathrm{~m}, 15 \mathrm{H}, \mathrm{Al}-\mathrm{H}), 2.06\left(\mathrm{~m}, 2 \mathrm{H}, \mathrm{CH}_{2}-\mathrm{CH}_{2}-\mathrm{CH}_{2}\right)$, $4.29\left(\mathrm{~m}, 2 \mathrm{H}, \mathrm{N}-\mathrm{CH}_{2}-\right), 4.39\left(\mathrm{~m}, 6 \mathrm{H}, \mathrm{O}-\mathrm{CH}_{2}-\right), 5.11$ (dd, ${ }^{3} \mathrm{~J}=8.63$ $\mathrm{Hz}$ and $\left.{ }^{2} J=1.38 \mathrm{~Hz}, 3 \mathrm{H}, \mathrm{CH}=\mathrm{C}\right), 5.26\left(\mathrm{dd},{ }^{3} J=14.15 \mathrm{~Hz}\right.$ and ${ }^{2} J$ $=1.5 \mathrm{~Hz}, 3 \mathrm{H}, \mathrm{CH}=\mathrm{C}), 5.91\left(\mathrm{~m}, 3 \mathrm{H},=\mathrm{CH}-\mathrm{CH}_{2}\right), 6.56(\mathrm{~m}, 2 \mathrm{H}, \mathrm{Ar}-$ $H), 7.04(\mathrm{~m}, 1 \mathrm{H}, \mathrm{Ar}-H), 7.42(\mathrm{~s}, 1 \mathrm{H},-\mathrm{C}=\mathrm{C} H-\mathrm{N}-) .{ }^{13} \mathrm{C}-\mathrm{NMR}$ $\left(\mathrm{CDCl}_{3}, \delta_{\mathrm{C}}, \mathrm{ppm}\right): 7.41(\mathrm{~d}) ; 14.19(\mathrm{z}) ; 23.29(\mathrm{k}) ; 23.97(\mathrm{e}) ; 26.16$ (o); 27.17 (r); 29.58 (s); 32.91 (j); 37.85 (n); 39.35 (q); 43.28 (p); 47.27 (m); 48.47 (l); 52.31 (f); 63.77 (c); 82.29 (i); 112.77 (w); 115.15 (a); 115.30 (y); 121.40 (g); 126.22 (v); $132.00(\mathrm{u}) ; 136.21$ (b); $137.96(\mathrm{t}) ; 153.39(\mathrm{x}) ; 153.75(\mathrm{~h})$.

Alkyne end-functionalized poly(pentafluorostyrene) (9). Alkyne functionalised poly(pentafluorostyrene) was synthesized according to a recently published procedure ${ }^{27}$ by atom transfer radical polymerization (ATRP) of 2,3,4,5,6-pentafluorostyrene in bulk initiated with 3-bromo-1-(trimethylsilyl)-1-propyne. $T_{\mathrm{g}}=$ $97{ }^{\circ} \mathrm{C}$. IR $\left(\mathrm{cm}^{-1}\right)$ : 3316 (C $\equiv \mathrm{C}-\mathrm{H}$ stretch); 2960 (C-H stretch); 1496 (F-aromatic stretch); 981 (C-F-aromatic bend). ${ }^{1} \mathrm{H}$ NMR $\left(\mathrm{CDCl}_{3}, \delta_{\mathrm{H}}, \mathrm{ppm}\right): 1.70-3.00\left(\mathrm{CH}_{2}-\mathrm{C} \equiv+\mathrm{CH}_{2}-\mathrm{CH}+\mathrm{H}-\mathrm{C} \equiv \mathrm{C}\right), 4.8$ (m, $1 \mathrm{H}, \mathrm{CH}-\mathrm{Br}) .{ }^{13} \mathrm{C}-\mathrm{NMR}\left(\mathrm{CDCl}_{3}, \delta_{\mathrm{C}}, \mathrm{ppm}\right): 16.56(\mathrm{i}) ; 32.12(\mathrm{j})$; 37.13-38.67 (k); 69.24 (g); 81.79 (h); 135.81 (l); 139.18 (n); 143.15 (o); $146.45(\mathrm{~m})$. SEC (THF): $\bar{M}_{\mathrm{n}}=3300 \mathrm{Da}, \bar{M}_{\mathrm{w}} / \bar{M}_{\mathrm{n}}=1.4$.

4-Poly(pentafluorostyrenyl)-1-(3-(tris(allyloxy)silyl)propyl-1H1,2,3-triazole (10). The alkyne end-functional polymer 9 (0.6 g, $0.18 \mathrm{mmol})$ and $\mathrm{CuI}(0.031 \mathrm{~g}, 0.162 \mathrm{mmol})$ were mixed in THF $(4 \mathrm{ml})$ followed by the addition of $2(0.062 \mathrm{~g}, 0.216 \mathrm{mmol})$ and $\mathrm{Et}_{3} \mathrm{~N}(0.1 \mathrm{ml}, 0.72 \mathrm{mmol})$. The click reaction proceeded at $35{ }^{\circ} \mathrm{C}$ for $20 \mathrm{~h}$. The product was precipitated in methanol and dried in vacuo. Yield: $0.59 \mathrm{~g}(89 \%) . T_{\mathrm{g}}=112{ }^{\circ} \mathrm{C}$. IR $\left(\mathrm{cm}^{-1}\right): 2960-2870$ (C-H stretch); 1653 (C=C stretch); 1498 (F-aromatic stretch); 981 (C-F-aromatic bend). ${ }^{1} \mathrm{H}$ NMR $\left(\mathrm{CDCl}_{3}, \delta_{\mathrm{H}}, \mathrm{ppm}\right): 0.70(\mathrm{Si}-$ $\left.\mathrm{CH}_{2}\right), 1.70-3.00\left(\mathrm{Si}-\mathrm{CH}_{2}-\mathrm{CH}_{2}+\mathrm{CH}_{2}-\mathrm{CH}+\mathrm{CH}=\mathrm{C}(\mathrm{N})-\mathrm{CH}_{2}\right), 4.25$ $\left(\mathrm{Si}-\mathrm{O}-\mathrm{CH}_{2}+\mathrm{Si}-\mathrm{CH}_{2}-\mathrm{CH}_{2}-\mathrm{CH}_{2}-\mathrm{N}\right), 5.15\left(\mathrm{CH}_{2}=\mathrm{CH}\right), 5.87\left(\mathrm{CH}_{2}=\right.$ $\mathrm{CH}), 7.60\left(\mathrm{~N}-\mathrm{CH}=\mathrm{C}(\mathrm{N})-\mathrm{CH}_{2}\right) \cdot{ }^{13} \mathrm{C}-\mathrm{NMR}\left(\mathrm{CDCl}_{3}, \delta_{\mathrm{C}}, \mathrm{ppm}\right): 7.51$ (d); 24.07 (e); 25.52 (i); 32.12 (j); 37.05-38.79 (k); 52.85 (f); 63.26 (c); 114.15 (a); 125.27 (g); 135.93 (l); 136.11 (b); 139.32 (n); 143.42 (o); $146.24(\mathrm{~m}) ; 153.16(\mathrm{~h}) . \bar{M}_{\mathrm{n}}=3300 \mathrm{Da}, \bar{M}_{\mathrm{w}} / \bar{M}_{\mathrm{n}}=1.4$.

\section{Procedure for stability measurements}

The hydrolytic stability of the Si-O-C linkage in 2 was determined using a previously described method. ${ }^{28} 30 \mathrm{mg}$ of sample was dissolved in $0.8 \mathrm{ml} \mathrm{CDCl}_{3}$ in an NMR tube to which $50 \mu \mathrm{l}$ $\mathrm{D}_{2} \mathrm{O}$ was added. The ${ }^{1} \mathrm{H}$-NMR of the mixture was recorded over time for $72 \mathrm{~h}$. The silyl ether cleavage was evaluated by integration and comparison of the $\mathrm{O}-\mathrm{CH}_{2}$ peak and the $\mathrm{CH}_{2}-\mathrm{Si}$ peak.

\section{Preparation of polymer films}

Cross-linkers (2, 4-8 and 10) (0.043 mmol) were dissolved in dry THF $(0.3 \mathrm{ml})$ and mixed with a 16 -functional cross-linker (VDT431) (0.0027 mmol) and hydride-terminated PDMS (DMS-H31) $(0.086 \mathrm{mmol})$. The platinum cyclovinylmethyl siloxane complex catalyst (511) (10-30 ppm) in dry THF was thereafter added and the mixture was mixed on a vortex mixer for 2 minutes. The mixture was thereafter degassed in vacuo to remove solvent and air bubbles. The mixture was poured into $1 \mathrm{~mm}$ thick steel molds on a fluorinated ethylene propylene (FEP) release liner and cured at $60{ }^{\circ} \mathrm{C}$ for $24 \mathrm{~h}$.

\section{Swelling experiments}

The gel fractions were determined using two methods. The first method, Soxhlet extraction with chloroform for 48 hours, was used to pull out all extractables. The samples were thereafter dried for $24 \mathrm{~h}$ and the gel fraction was determined as the weight after extraction and drying $\left(m_{\mathrm{e}}\right)$ to the initial weight of the sample $\left(m_{0}\right)$ as $W_{\text {gel }}=m_{\mathrm{e}} / m_{0}$.

The second method consisted of swelling experiments used to pull out leachables from the prepared films. Samples were swelled in chloroform for 48 hours at RT. The solvent was replaced after 24 hours. The solvent was then decanted and the films were washed several times with chloroform. The samples were thereafter dried for $24 \mathrm{~h}$. Gel fractions were determined as the weight after extraction and drying $\left(m_{\mathrm{e}}\right)$ to the initial weight of the sample $\left(m_{0}\right)$ as $W_{\text {gel }}=m_{\mathrm{e}} / m_{0}$.

\section{Results and discussion}

The azide functional, silicone compatible vinyl cross-linker (2) was synthesized in two steps via the silyl ether reaction between (3-bromopropyl)trichlorosilane and allyl alcohol and subsequent substitution of bromine with azide as illustrated in Scheme 1.

The azide cross-linker was isolated as an orange-yellow oil with a high yield and high purity. The vinyl cross-linker proved to be hydrolytically stable under the utilized synthesis conditions which included several extraction procedures with water. The stability of the formed silyl ether bond was evaluated using ${ }^{1} \mathrm{H}$-NMR in $\mathrm{D}_{2} \mathrm{O}$. Spectra were recorded over time and integration of the $\mathrm{O}-\mathrm{CH}_{2}$ peak compared to integration of other peaks indicated that no detectable cleavage of the $\mathrm{Si}-\mathrm{O}-\mathrm{C}$ bond had taken place in the stability test during the 72 hours.

The alkyne functional molecules used in the click reactions with 2 are shown in Table 1. A general reaction scheme for the click reaction is illustrated in Scheme 2.

The click reaction was used to prepare a variety of different functional cross-linkers as shown in Table 1. Two of the employed alkynes, $\mathbf{3}$ and $\mathbf{9}$, were not commercially available but prepared for this study. 3 was prepared through a Williamson ether synthesis of 4-methylumbelliferone and propargyl bromide using $\mathrm{K}_{2} \mathrm{CO}_{3}$ as the base catalyst. 9 was polymerized under controlled ATRP conditions using a previously published procedure ${ }^{27}$ yielding an alkyne functional poly(pentafluorostyrene). The functionalized cross-linkers were all prepared under similar click reaction conditions in $\mathrm{THF}$ using a CuI- $\mathrm{Et}_{3} \mathrm{~N}$ catalytic system. In the case

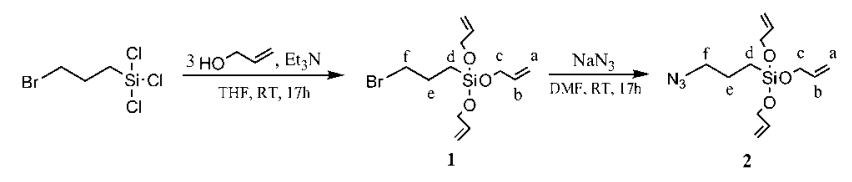

Scheme 1 Preparation of the azide-functional vinyl cross-linker 2. 
Table 1 Alkynes and functional cross-linkers from click reactions. Letters are used in assignment of ${ }^{13} \mathrm{C}-\mathrm{NMR}$ spectra

Alkyne Click-product
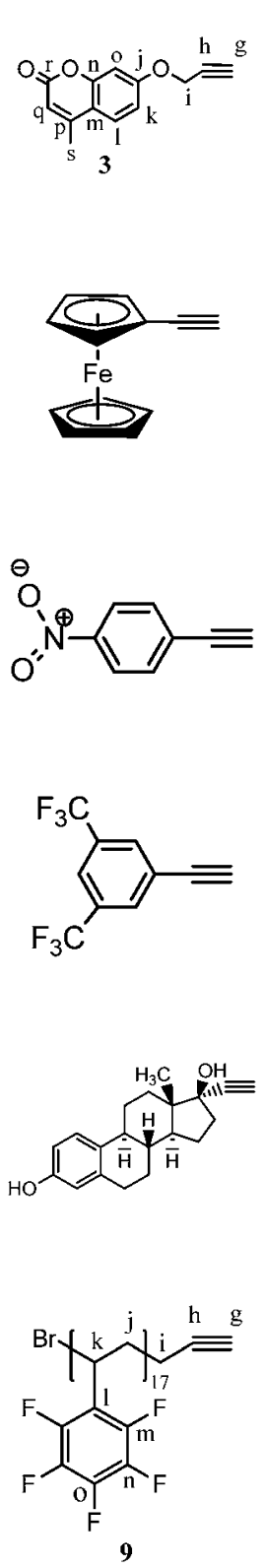

9
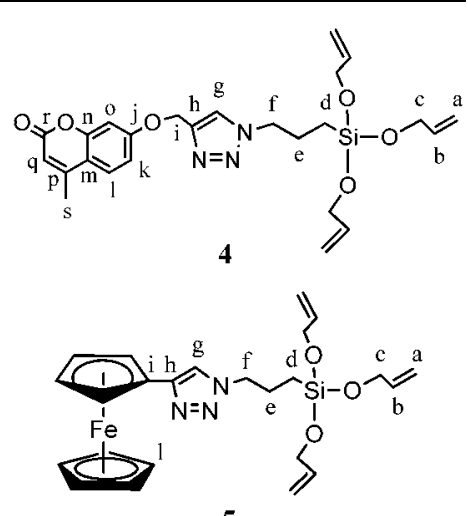

5
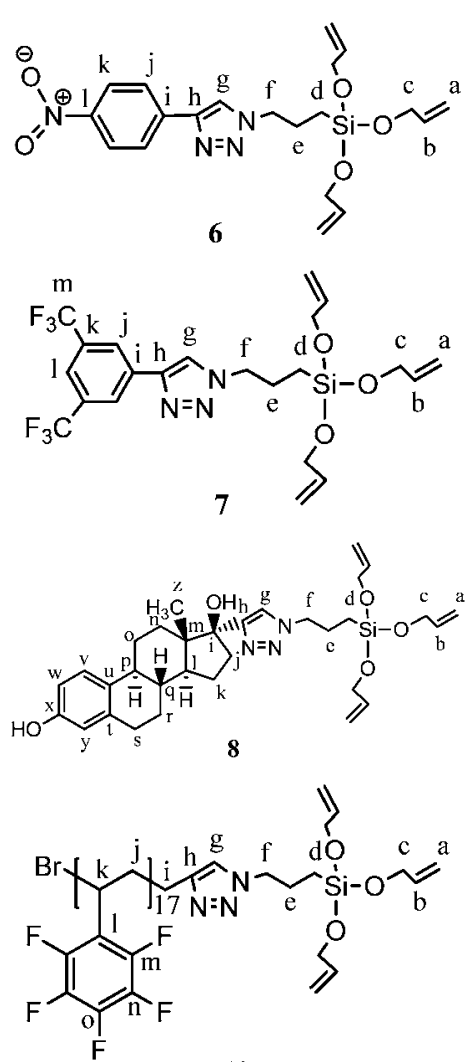

10

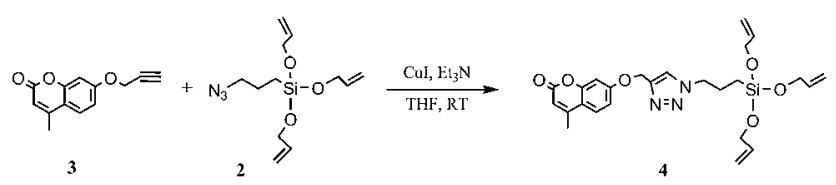

Scheme 2 General reaction scheme for click reaction between the azide crosslinker (2) and alkyne exemplified by $\mathbf{3}$.

of 5, 8 and 10 yields were high ( $\sim 90 \%)$ but in the case of 4 and 6 the yields were in the order of $50 \%$. This was assessed to be acceptable and was not attempted optimized.
The synthesized alkynes and all novel vinyl cross-linkers, 4-8 and 10, were thoroughly characterized by FTIR and ${ }^{1} \mathrm{H}$ - and ${ }^{13} \mathrm{C}-$ NMR. FTIR was used to confirm the completion of the click reactions by the disappearances of the alkyne band at approximately $3300 \mathrm{~cm}^{-1}$ and the very distinct azide band at approximately $2100 \mathrm{~cm}^{-1}$. The presence of the triazole proton in ${ }^{1} \mathrm{H}$ NMR points to the successful formation of reaction products. The triazole proton appears from $\delta_{\mathrm{H}}=7.42 \mathrm{ppm}$ to $\delta_{\mathrm{H}}=8.30$ ppm depending on the electron density of the triazole. Representative spectra can be found as ESI. $\uparrow$ If small residues of azide/alkyne can be detected, they are easily removed by use of resins which facilitates a simple purification procedure. In connection with the synthesis of 7 , a small alkyne band was detected by FTIR and a peak for the alkyne proton was also detected by ${ }^{1} \mathrm{H}-\mathrm{NMR}$ at $\delta_{\mathrm{H}}=3.26 \mathrm{ppm} .7$ was consequently treated with an azide functional Merrifield resin in order to remove the residual alkyne reactant by additional click reaction and resulting attachment of the alkyne reactant to the resin. The azide resin with the excess alkyne attached could thereafter be removed from the reaction mixture by simple filtration.

The PDMS networks were prepared from cross-linkers 2, 4-8 and 10, hydride terminated PDMS and a platinum catalyst according to Scheme 3 .

A commercially available 16-functional silicone vinyl crosslinker was used to reinforce the network since networks prepared solely from three-functional cross-linkers are known to be very soft and have low mechanical breakdown strength. ${ }^{29}$ The molar ratio between the three-functional synthesized crosslinkers and the 16-functional commercial silicone cross-linker were $16: 1$ to ensure a high concentration of the functionalized cross-linkers and minimize possible competition from the 16functional cross-linker. The networks were prepared with equimolar amounts, meaning that the number of functional hydride groups of the polymer corresponds to the total number

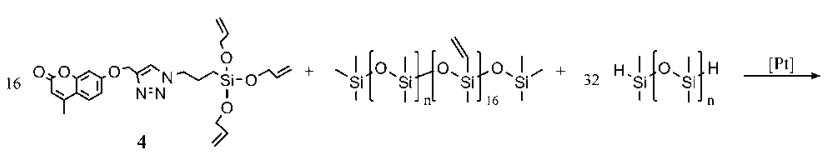

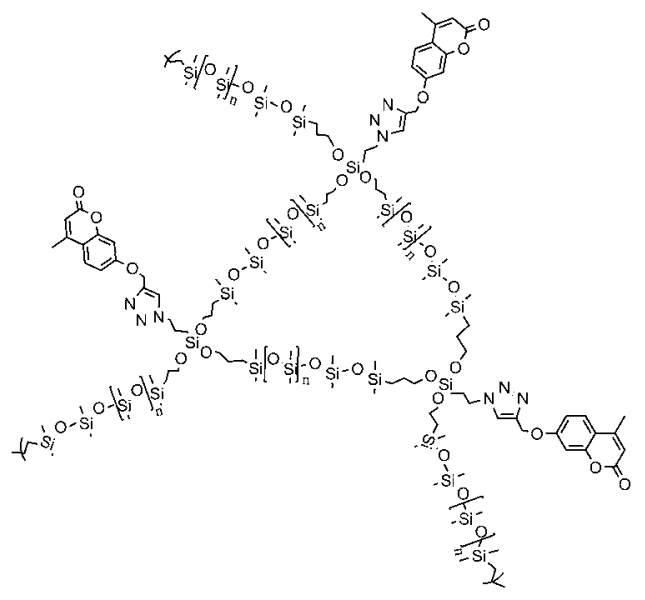

Scheme 3 Cross-linking reaction between 4 and hydride terminated PDMS. 
of vinyl groups on the two cross-linkers. The PDMS films ranged from light yellow in color for films with 6 and 8 to light brown for films with 5 and 7 and dark brown for 10. The film with 2 was completely transparent whereas the film with $\mathbf{4}$ was light grey and opaque. Images of all films can be found in the ESI. $†$

All prepared films were characterized by FTIR. There was, however, no differences between the films with different functional cross-linkers since the concentrations of cross-linkers in the films were found to be too low to be detected by this technique. A solid state ${ }^{13} \mathrm{C}$-NMR spectrum of the elastomer with 4 obtained over $20 \mathrm{~h}$ reveals the dominating $\mathrm{Si}-\mathrm{CH}_{3}$ resonance at $\sim 1 \mathrm{ppm}$. In addition three small resonances at $\sim 4, \sim 8, \sim 24 \mathrm{ppm}$ can be assigned to $\mathrm{CH}_{2}$ close to $\mathrm{Si}$ atoms. Weak signals in the 100-160 ppm range indicate the presence of aromatic carbons. The solid state ${ }^{13} \mathrm{C}-\mathrm{NMR}$ analysis thus strongly supports the presence of the silyl-based cross-linker in the network.

Swelling and extraction experiments were used to determine the gel fraction of the films. This was done in order to further elucidate if the synthesized cross-linkers had reacted with the PDMS chains and were incorporated covalently in the network. Possible problems that such modified networks could encounter include poor compatibility with the matrix and steric hindrance of the three-functional cross-linker leading to dangling chains and extractable substructures containing primarily the triazole cross-linkers. Some of the produced films had indeed large sol fractions especially from the Soxhlet extraction data (Table 2). It is, however, not common practice to perform this type of extractions when investigating PDMS network gel fractions as it is a very aggressive method. The swelling experiments performed are therefore more suitable for comparison with previously performed PDMS swelling experiments. ${ }^{30,31}$ Optimized PDMS elastomers usually have gel fractions of $95-97 \%$ whereas stoichiometrical PDMS networks usually have gel fractions around $90 \%$. This can be explained by the optimized PDMS elastomers having a stoichiometry of 1.1-1.4 which means that the cross-linker is in excess and that all cross-linker sites do not need to react thus reducing steric hindrance. ${ }^{32}$ The networks prepared in this study are all stoichiometric such that gel fractions of less than $90 \%$ are anticipated.

A visual evaluation of the dry sol and gel fraction revealed that all films (gel fractions) retained their initial colors which are due to the presence of the functional cross-linkers. Furthermore all sol fractions bore little or no evidence of color from cross-linkers. In order to further investigate the nature of the gel and sol

Table 2 Gel contents of samples as results from Soxhlet extractions and swelling experiments (\%)

\begin{tabular}{lll}
\hline Cross-linker & $\begin{array}{l}\text { Gel fraction } \\
\text { (Soxhlet extraction) }\end{array}$ & $\begin{array}{l}\text { Gel fraction } \\
\text { (swelling) }\end{array}$ \\
\hline $\mathbf{2}$ & 78.5 & 84.1 \\
$\mathbf{4}$ & 57.3 & 92.2 \\
$\mathbf{5}$ & 52.8 & 82.7 \\
$\mathbf{6}$ & 56.8 & 68.5 \\
$\mathbf{7}$ & 74.1 & 90.1 \\
$\mathbf{8}$ & 55.9 & 59.3 \\
$\mathbf{1 0}$ & 41.5 & 69.1
\end{tabular}

fraction, the fluorescence intensity was measured of both the dried sol and the gel fraction of the film prepared with the fluorescence cross-linker 4 after the Soxhlet extraction experiment. The fluorescence intensity was also measured for the film with 4 before the Soxhlet extraction. Results are shown in Fig. 1.

It was observed that the fluorescence intensity of the film prepared with 4 only decreases by $4 \%$ after extraction. This indicates that cross-linker $\mathbf{4}$ is indeed incorporated into the PDMS network gel fraction and is thus covalently bound to the network. The sol fraction exhibits very low fluorescence intensity and therefore most likely consists of PDMS substructures not connected to the network such as inert or mono-functional PDMS chains. It can hence be concluded, that the synthesized crosslinkers can be successfully incorporated into PDMS networks.

To investigate the distribution of the grafted functionalities, fluorescence microscopy was performed on the film prepared with cross-linker 4 which acts as a fluorescent tag in the network as seen in Fig. 2.

It is seen that the film with $\mathbf{4}$ is indeed fluorescent and that the fluorescent cross-linker is evenly distributed throughout the network. This indicates that the synthesized triazole crosslinkers can successfully be incorporated into PDMS networks in a well distributed manner.

Mechanical characterization was performed by determining the storage and loss moduli for all films. In Fig. 3 the results for films prepared with $\mathbf{2}$ and $\mathbf{4}$ are presented. A similar plot for films with 5-8 and $\mathbf{1 0}$ can be found in the ESI. $\dagger$

It is seen in Fig. 3 that both networks are well cross-linked as the storage modulus for both networks are of an order of 10 to 100 times that of the loss modulus for the investigated frequency regime. ${ }^{32}$ The elastomer containing cross-linker 4 is seen to have slightly lower moduli than the film with crosslinker 2. This can be explained by the bulkier group attached on 4 which thereby creates a small diluting effect in the network creating a less elastic film. All other films showed similar mechanical behavior. The elastomer with cross-linker $\mathbf{1 0}$ has lower moduli than the other films. This corresponds with the results seen from the extraction experiments which showed that the film with $\mathbf{1 0}$ has a significantly smaller gel fraction than the other networks. The large poly(pentafluorostyrene) groups thereby creates a soft network with a large portion of extractable

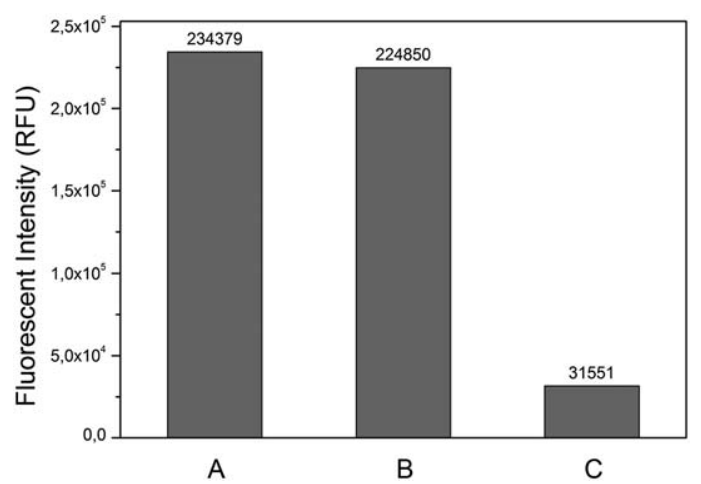

Fig. 1 Fluorescent intensity of A: film with $\mathbf{4}$ before Soxhlet extraction; B: dried film with $\mathbf{4}$ after Soxhlet extraction (gel fraction) and C: dried sol fraction of film with $\mathbf{4}$. 


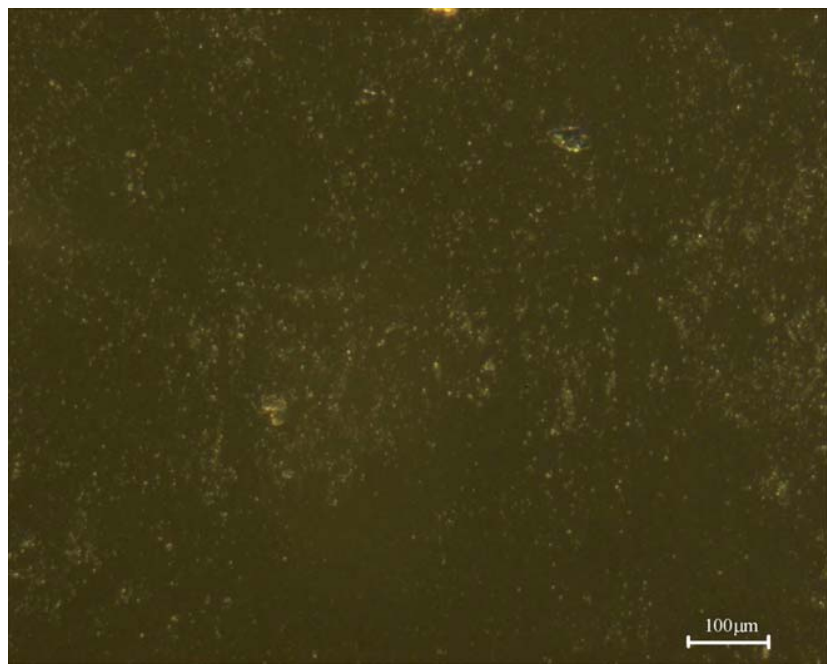

Fig. 2 Fluorescent microscopy image of the film prepared with cross-linker 4.

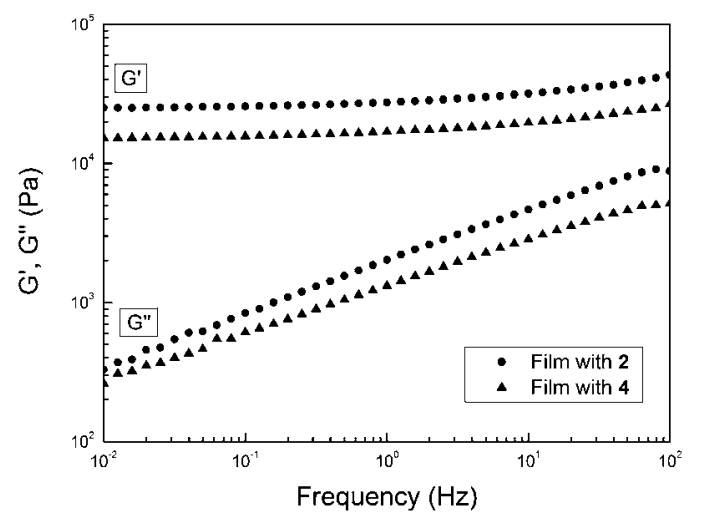

Fig. 3 Storage modulus $\left(G^{\prime}\right)$ and loss modulus $\left(G^{\prime \prime}\right)$ as functions of frequency for films prepared with $\mathbf{2}$ and $\mathbf{4}$.

substructures. This can be due to incompatibility between the two polymers leading to phase separation which is also visually evident from the darker domains formed in the film.

Thermal gravimetric analysis (TGA) was used to investigate the effects of the different functional cross-linkers on the thermal stability of the PDMS films (Fig. 4).

All TGA curves show a two step mass loss process. The first degradation step occurring from $390{ }^{\circ} \mathrm{C}$ to $540{ }^{\circ} \mathrm{C}$ (mass loss of 1.8-12.7 wt\%) depending on functionality corresponds to the cleavage of $\mathrm{Si}-\mathrm{CH}_{3}$ bonds. In the case of $\mathbf{1 0}$ it is noted that at 510 ${ }^{\circ} \mathrm{C}$ all the poly(pentafluorostyrene) has degraded..$^{33}$ The second decomposition step occurring from $5900^{\circ} \mathrm{C}$ to $690{ }^{\circ} \mathrm{C}$ (mass loss of 32.5-86.4 wt\%) can be attributed to structural rearrangements followed by mineralization of the material. ${ }^{34}$ The decomposition temperatures are taken as the peak temperature of the first derivate of the temperature with time. The TGA curves show that the PDMS samples have different decomposition temperatures depending on the incorporated functionality with $\mathbf{5}$ giving the highest initial degradation temperature $\left(540{ }^{\circ} \mathrm{C}\right)$ due to higher thermal stability of ferrocene ${ }^{35}$ which increases the degradation temperature of the PDMS film. It is

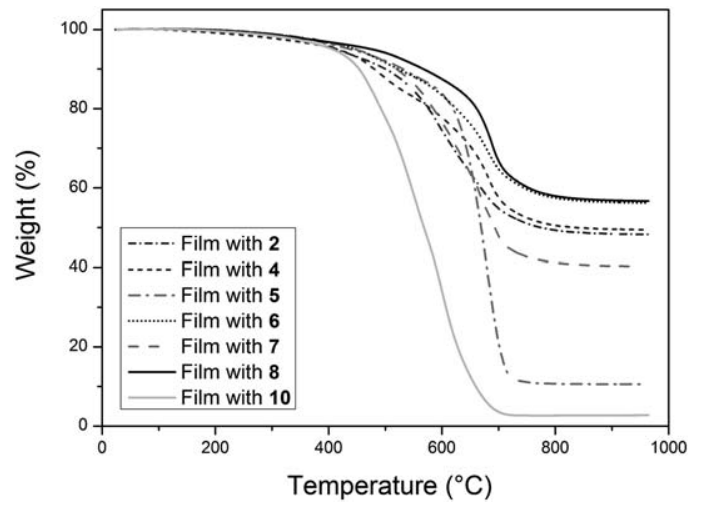

Fig. 4 TGA measurements of PDMS films prepared with cross-linkers 2, 4-8 and $\mathbf{1 0}$.

hypothesized that the ferrocene cross-linker, however, also catalyzes the PDMS degradation such that mineralization occurs to a larger extent. The catalytic effect can also be observed for the film with $\mathbf{1 0}$ (poly(pentafluorostyrene)). It is moreover noted that all films with a functional cross-linker except for 10 induced a higher decomposition temperature than films with a non-functional cross-linker (2).

The dielectric properties were determined by dielectric relaxation spectroscopy (DRS) for the films prepared with the 2 and 6 cross-linkers, see Fig. 5.

It was found, that the dipolar nature of the 6 cross-linker, due to the nitro group, increases the dielectric properties of the film from 2.3 for films with 2 to 3.1 for films with 6 which corresponds to an increase of $35 \%$. This increase is remarkable since the content of this dipolar cross-linker is only around 0.75 $\mathrm{wt} \%$ corresponding to $0.25 \mathrm{wt} \%$ of the pure dipole molecule. It is therefore likely that by increasing the amount of dipolar cross-linker it would be possible to achieve a large increase in the dielectric permittivity which could improve the performance of dielectric elastomers to a very great extent.

The impact of the fluorine containing cross-linkers on the release properties of the PDMS films was determined using contact angle measurements. The contact angle was found to increase from $108^{\circ} \pm 2.5$ for the film with cross-linker 2 to

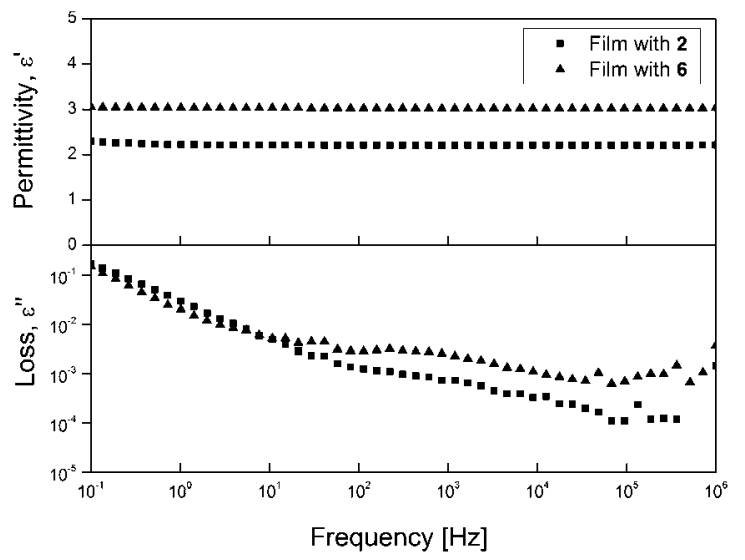

Fig. 5 Permittivity spectra of films prepared with the $\mathbf{2}$ and $\mathbf{6}$ cross-linkers showing both the frequency dependence on the permittivity $\left(\varepsilon^{\prime}\right)$ and the dielectric loss $\left(\varepsilon^{\prime \prime}\right)$. 
$116^{\circ} \pm 2.5$ for the PDMS film with cross-linker 10. This is due to the hydrophobic nature of the poly(pentafluorostyrene) which causes a migration of the fluorinated polymer to the PDMS surface $^{36}$ and thus increasing the contact angle and thereby also a possible improvement of release properties for PDMS films during processing. ${ }^{8}$ The film with 7 showed no increase in contact angle compared to the film with 2 . This is probably due to the low concentration of fluorine on this cross-linker which allows the cross-linker to be encapsulated within the PDMS network and no migration to the surface will consequently occur.

\section{Conclusions}

A novel silicone compatible cross-linker that allows for click reactions and thereby grafting of various molecular functionalities into PDMS networks has been developed. The developed system opens up for a wide variety of possible reactions and network functionalizations which can expand the application area of PDMS networks greatly. The functionalization of PDMS networks was demonstrated by the successful synthesis of a number of diverse functional cross-linkers that were used to create PDMS films. It was shown that all functional cross-linkers had been successfully incorporated into the network and that only minute amounts of the functionality could be extracted from the network. It was also demonstrated by fluorescence microscopy that the functional cross-linkers were well distributed within the PDMS network. Thermal gravimetric analysis (TGA) showed that the incorporation of a ferrocene functionality increased the thermal degradation temperature of the PDMS network. Furthermore it was shown that the incorporation of a dipolar cross-linker could improve the dielectric permittivity of PDMS by a factor of $35 \%$ at only $0.25 \mathrm{wt} \%$ of dipole-functionality and that the contact angle of the PDMS films could be increased from $108^{\circ}$ to $116^{\circ}$ by the incorporation of a small poly(pentafluorostyrene) chain.

\section{Acknowledgements}

The authors wish to express thanks to Morten Bjerring, Aarhus University for help with solid state NMR and acknowledge the Danish National Advanced Technology Foundation for financial support.

\section{Notes and references}

1 E. G. Rochow, in Silicon-Based Polymer Science - A Comprehensive Resource, ed. J. M. Zeigler and F. W. G. Fearon, American Chemical Society, 1990, pp. xiii-xxii.

2 J. de Jong, R. G. H. Lammertink and M. Wessling, Lab Chip, 2006, 6, 1125-1139.

3 M. K. Jensen, A. Bach, O. Hassager and A. L. Skov, Int. J. Adhes. Adhes., 2009, 29, 687-693.

4 P. Brochu and Q. Pei, Macromol. Rapid Commun., 2010, 31, 10-36. 5 J. E. Mark, in Silicon-based Polymer Science: A Comprehensive Resource, ed. J. M. Zeigler and F. W. G. Fearon, American Chemical Society, 1990, pp. 47-68.

6 F. Carpi, G. Gallone, F. Galantini and D. De Rossi, Adv. Funct. Mater., 2008, 18, 235-241.
7 B. Li, S. Chen and J. Zhang, Polym. Chem., 2012, 3, 2366-2376. 8 S. Vudayagiri, M. D. Junker and A. L. Skov, Polym. J., 2012, DOI: $10.1038 /$ pj.2012.227.

9 H. C. Kolb, M. G. Finn and K. B. Sharpless, Angew. Chem., Int. Ed., 2001, 40, 2004-2021.

10 P. Wu, A. K. Feldman, A. K. Nugent, C. J. Hawker, A. Scheel, B. Voit, J. Pyun, J. M. J. Fréchet, K. B. Sharpless and V. V. Fokin, Angew. Chem., Int. Ed., 2004, 43, 3928-3932.

11 M. Meldal, Macromol. Rapid Commun., 2008, 29, 1016-1051. $12 \mathrm{~W}$. H. Binder and R. Sachsenhofer, Macromol. Rapid Commun., 2008, 29, 952-981.

13 A. D. Thomsen, E. Malmström and S. Hvilsted, J. Polym. Sci., Part A: Polym. Chem., 2006, 44, 6360-6377.

14 A. E. Daugaard, S. Hvilsted, T. S. Hansen and N. B. Larsen, Macromolecules, 2008, 41, 4321-4327.

15 S. Hvilsted, Polym. Int., 2012, 61, 485-494.

16 F. Gonzaga, G. Yu and M. A. Brook, Macromolecules, 2009, 42, 9220-9224.

17 F. Gonzaga, G. Yu and M. A. Brook, Chem. Commun., 2009, 1730-1732.

18 U. Schmidt, P. C. Zehetmaier and B. Rieger, Macromol. Rapid Commun., 2010, 31, 545-548.

19 S. Halila, M. Manguian, S. Fort, S. Cottaz, T. Hamaide, E. Fleury and H. Driguez, Macromol. Chem. Phys., 2008, 209, 1282-1290.

20 B. Kussmaul, S. Risse, G. Kofod, R. Waché, M. Wegener, D. N. McCarthy, H. Krüger and R. Gerhard, Adv. Funct. Mater., 2011, 21, 4589-4594.

21 R. D. A. Hudson, J. Organomet. Chem., 2001, 637-639, 47-69.

22 P. Köpf-Maier, H. Köpf and E. W. Neuse, J. Cancer Res. Clin. Oncol., 1984, 108, 336-340.

23 I. Javakhishvili and S. Hvilsted, Polym. Chem., 2010, 1, 1650-1661. 24 M. Coates, S. Griveau, F. Bedioui and T. Nyokong, Electroanalysis, 2012, 24, 1833-1838.

25 E. C. Dreaden, S. C. Mwakwari, Q. H. Sodji, A. K. Oyelere and M. A. El-Sayed, BioconjugateChem., 2009, 20, 2247-2253.

26 J. A. Opsteen and J. C. M. van Hest, Chem. Commun., 2005, 57-59. 27 I. Dimitrov, S. Takamuku, K. Jankova, P. Jannasch and S. Hvilsted, Macromol. Rapid Commun., 2012, 33, 1368-1374.

28 D. B. Thompson, F. Gonzaga, A. S. Fawcett and M. A. Brook, Silicon Chem., 2008, 3, 327-334.

29 A. G. Bejenariu, H. K. Rasmussen, A. L. Skov, O. Hassager and S. M. Frankaer, Rheol. Acta, 2010, 49, 807-814.

30 S. M. G. Frankær, M. K. Jensen, A. G. Bejenariu and A. L. Skov, Rheol. Acta, 2012, 51, 559-567.

31 J. N. Lee, C. Park and G. M. Whitesides, Anal. Chem., 2003, 75, 6544-6554.

32 A. L. Larsen, K. Hansen, P. Sommer-Larsen, O. Hassager, A. Bach, S. Ndoni and M. Jørgensen, Macromolecules, 2003, 36, 10063-10070.

33 K. Jankova and S. Hvilsted, Macromolecules, 2003, 36, 1753-1758. 34 G. Camino, S. M. Lomakin and M. Lageard, Polymer, 2002, 43, 2011-2015.

35 A. S. Abd-El-Aziz and E. K. Todd, Coord. Chem. Rev., 2003, 246, 3-52.

36 S. Borkar, K. Jankova, H. W. Siesler and S. Hvilsted, Macromolecules, 2004, 37, 788-794. 